\title{
Steering in computational science: mesoscale modelling and simulation
}

\author{
J. Chin, J. Harting, S. Jha, P. V. Coveney ${ }^{1}$ \\ Centre for Computational Science \\ Christopher Ingold Laboratories \\ University College London \\ 20 Gordon Street \\ London WC1H OAJ \\ U.K.
}

\author{
A. R. Porter, S. M. Pickles \\ Manchester Computing \\ Kilburn Building \\ The University of Manchester \\ Oxford Road \\ Manchester M13 9PL \\ U.K.
}

October 30, 2018

\begin{abstract}
This paper outlines the benefits of computational steering for high performance computing applications. Lattice-Boltzmann mesoscale fluid simulations of binary and ternary amphiphilic fluids in two and three dimensions are used to illustrate the substantial improvements which computational steering offers in terms of resource efficiency and time to discover new physics. We discuss details of our current steering implementations and describe their future outlook with the advent of computational grids.
\end{abstract}

\footnotetext{
${ }^{1}<$ P.V.Coveney@ucl.ac.uk $>$
} 


\section{Introduction}

Many phenomena in condensed matter physics operate at length and time scales which are too large for detailed microscopic modelling. In microscopic models, based on classical molecular dynamics, usually every atom or molecule within a system is considered, resulting in rapidly increasing complexity for increasing problem sizes. This tends to restrict the currently treatable length scales to the order of several nanometres and the timescales to the order of nanoseconds since the computing power required for larger length and timescales is unavailable today. For example, a microscopic description of a fluid would track the position, momentum, and energy of every single fluid molecule. At room temperature, a microscopic model of a single cubic millimetre of monatomic gas would have to deal with around $10^{17}$ variables. Macroscopic models, on the other hand, usually deal with a smaller number of variables, often more closely related to physical observables. For example, a macroscopic model of a fluid would describe its velocity, density and temperature at various points.

Statistical mechanics is used to extract macroscopic, thermodynamic descriptions from the underlying microscopic representation. While standard methods exist for performing this contraction of description for systems at thermodynamic equilibrium [1], we shall be primarily interested in time-dependent, non-equilibrium systems for which there is less widespread agreement about their statistical mechanical description. In addition, comparatively few established methods exist for the treatment of complex systems which contain processes operating on several length and time scales. This situation presents a general problem for both microscopic and macroscopic models. Whereas in microscopic descriptions, a vast amount of computational effort is required to model mesoscopic systems spanning several length and time scales, macroscopic treatments omit fine details which may give rise to the characteristic behaviour of the system.

Because of the shortcomings of microscopic and macroscopic descriptions, and the tremendous importance of fluid dynamics, there is currently considerable interest in mesoscale models. These models coarse grain most of the atomic or molecular details but retain enough of the essential physics to describe the phenomena of interest. They are intended to treat systems at intermediate length scales between several nanometres and a few millimetres, and processes operating on multiple length and time scales. Examples of such systems can be found in everyday life: detergents, shampoos, milk, blood, and paint are materials whose macroscopic behaviour is induced by their microscopic and/or mesoscopic properties. A very descriptive example can be observed in the kitchen and can easily be reproduced by the reader: due to the microscopic interactions between starch molecules in cornflour, as the starch molecules jam into one another, a mixture of cornflour and water becomes more difficult to stir if one stirs it quickly.

Computer modelling forms a valuable tool in understanding the behaviour of mesoscale 
systems [2, 3, 4, 5], and much time and effort is currently being invested in such techniques. In this article, we provide a brief overview of a few such mesoscale modelling techniques, and focus in particular on the lattice-Boltzmann fluid dynamical method. We describe its implementation, and the problems which arise when the computer implementation is run 'statically', without interaction with either the user or other computational components. These problems may be solved by adding extra functionality which permits the interoperation of the lattice-Boltzmann code with other programs, such as separate code to monitor and readjust the simulation in real-time, or a user interface to permit a human to 'steer' the simulation as it runs. We give examples of two such functionalities: in one, the functionality of the simulation code is made available, through the use of a wrapper layer, to a high-level language, which permits versatile control of simulations; in the other, the code is connected to a general-purpose steering library which permits users to remotely control many kinds of simulation as they run.

Mesoscale simulations often require access to high-end computational and visualisation resources; we therefore proceed to discuss computational steering in such a general context, and how it may permit much more efficient use of such resources.

The literature on computational steering is quite substantial [6]. However, most papers [7, 8] have focussed on the design and architectural details of computational steering and then go on to give a prototype implementation of their steering system. Our paper represents a different approach, in that our motivation is a scientific problem for which computational steering is shown to be an effective tool. We do this by highlighting the advantages that computational steering brings over traditional non-steered simulations.

Computational grids are an increasingly popular paradigm of computation, somewhat akin to traditional distributed computing, yet with a major extension in that they enable the transparent sharing and collective use of resources (anything from spare PC CPUs to databases or high-end hardware), which would otherwise be individual and isolated facilities. Therefore, we discuss how the advent of computational grids is expected to considerably facilitate modelling with high-performance computers in general, and computational steering in particular.

\section{Mesoscale modelling and simulation methods}

Many mesoscale situations of interest involve fluids, particularly mixtures of fluids which exhibit complicated behaviour due to the interactions of their individual molecules. When attempting to model such systems, one must treat both the bulk flow, or hydrodynamic behaviour, and the interactions. Hydrodynamic behaviour is very difficult and expensive to treat by atomistic methods, but relatively straightforward to handle at the continuum level; conversely, the fluid interactions can be examined at the atomistic level, but are usually 
not straightforward to incorporate at the continuum level. There are some cases where interactions between microscopic particles may give rise to macroscopic flow behaviour for example, Marangoni flow [9], where gradients in surface tension (due to, for example, uneven distribution of surfactant at an interface[10]) induce macroscopically observable fluid flow.

Many mesoscale methods start by exploiting the surprising and convenient fact that it is not necessary to keep track of every single molecule of a fluid in order to reproduce its hydrodynamic behaviour. Instead, it is sufficient to group very large numbers of molecules into Lagrangian 'packets of fluid', and treat these packets as self-contained particles themselves; interactions can take place between these mesoscopic groupings of particles, rather than their constituent molecules. Provided that certain restrictions, such as isotropy and conservation of mass and momentum, are adhered to, then the resulting large-scale behaviour is extremely similar to, and can often be related to, that which would result from treating each molecule individually [11, 12].

The technique of dissipative particle dynamics (DPD) [13, 14] tracks the position $\mathbf{r}_{i}$ and momentum $\mathbf{p}_{i}$ of each mesoscopic particle $i$. The algorithm consists of two stages: in the first, particle positions are advected according to their momenta, so $\mathbf{r}_{i} \rightarrow \mathbf{r}_{i}+\mathbf{p}_{i} \delta t$. In the second stage, the momentum of each particle is updated according to the force acting upon it, so $\mathbf{p}_{i} \rightarrow \mathbf{p}_{i}+\sum_{j \neq i} \mathbf{F}_{i j}^{C}+\sum_{j \neq i} \mathbf{F}_{i j}^{D}+\sum_{j \neq i} \mathbf{F}_{i j}^{R}$, where $\mathbf{F}_{i j}^{C}$ is a conservative interaction force between different particles, $\mathbf{F}_{i j}^{D}$ is a dissipative, viscous force, and $\mathbf{F}_{i j}^{R}$ is a random force to introduce stochastic fluctuations. An extra force may be introduced to allow interactions between different particles [15].

While DPD permits a continuous range of values for $\mathbf{r}_{i}$ and $\mathbf{p}_{i}$, realistic models may be created which discretize the position, the momentum, or both. In the Lattice Gas Automaton (LGA) method [16, 17], mesoscopic particles are only permitted to occupy points on a Bravais lattice, discretizing space, and are only permitted to travel along the lattice vectors, discretizing momentum. The update algorithm also consists of two steps: during the "advection" step, particles travel along their velocity vectors to adjacent lattice sites; in the "collision" step, particles at each individual lattice site undergo collisions during which their velocities are redistributed in a manner which conserves the total mass and momentum at each site. Provided the lattice is carefully chosen to ensure isotropy of the fluid, the large-scale behaviour will be hydrodynamically correct. A substantial advantage of this algorithm is that it only requires Boolean operations, and that it is unconditionally numerically stable [18].

Fluid mixtures may be simulated by introducing different species onto the lattice, often denoted by colour: red particles may represent oil, and blue particles water. Interactions between the different species may then be introduced by imagining that particles carry a 'colour charge', and experience a force due to the colour field generated by surrounding particles [19]. 
The red and blue fluids may be made immiscible by introducing a force which compels red particles to travel up the colour-field gradient towards regions of higher red density, and compels blue particles to travel towards regions of higher blue density; a mixture of red and blue particles will then separate into separate single-colour regions.

Porous media may be simulated by blocking off some lattice sites: any particles which would travel into the blocked sites during the advection step are bounced back to travel in the opposite direction, producing a no-slip boundary. The flow of oil-water mixtures in porous media has been successfully modelled using this technique [20].

An amphiphilic particle, such as a detergent molecule, typically contains two parts: a water-loving head, and an oil-loving tail. The behaviour of such molecules can be very complicated. In a mixture of oil and water, such a particle will seek out regions of oilwater interface, and reduce the interfacial tension. Solutions of amphiphile in water may spontaneously assemble to produce a variety of different phases, ranging from simple spherical or wormlike clusters called "micelles", to extensive sponge-like phases [21].

The 'coloured particle' description may be extended to cover the case of amphiphiles, by introducing a new species of mesoscopic particle, which has an orientational degree of freedom. A single such mesoscopic particle can be regarded as consisting of a red particle and a blue particle bolted together, so that the whole particle possesses no net colour charge, but will tend to align itself with the colour field; the orientation of the mesoscopic particle represents some sort of average of the orientations of its constituent molecules. Lattice gas models with amphiphile particles have been used to simulate the effect of surfactants on oil-water mixtures in porous media [22], and the self-assembly of micelles [23, 24].

In the model proposed by Malevanets and Kapral [25], sometimes called the 'Realcoded Lattice Gas', or 'Discrete Simulation Automaton' (DSA) model, the mesoscopic particles occupy discrete cells in space, but are permitted to have real-valued velocities. During the collision step of the algorithm, the velocities of the particles in each cell are transformed according to

$$
\mathbf{v}_{i} \rightarrow V+\omega\left[\mathbf{v}_{i}-V\right],
$$

where $\omega$ is a random rotation and $V$ the centre of mass velocity of particles in a cell. The total momentum in each cell remains unchanged, producing hydrodynamic behaviour, while the randomization of the velocities produces dissipative behaviour. Particles then travel along their velocity vectors to nearby cells. This model may be generalized to treat immiscible fluid mixtures [26] and amphiphiles [27] in much the same manner as with LGA models.

The lattice Boltzmann (LB) method is a simplification of LGA: particles have discretized positions and momenta, but rather than individual Boolean particles being tracked around a lattice, their real-valued population is stored, resulting in a less noisy method. We examine LB in more detail in Section 3 


\section{Lattice Boltzmann models of immiscible and amphiphilic fluids}

The lattice Boltzmann algorithm is a powerful method for simulating fluid flow. Much of this power lies in the ease with which boundary conditions can be imposed, and with which the model may be extended to describe mixtures of interacting complex fluids. Rather than tracking the state of individual atoms and molecules, as is done in molecular dynamics, or tracking individual discrete mesoscopic 'packets of fluid', as in LGA or DSA algorithms, the lattice Boltzmann method describes the dynamics of the singleparticle distribution function of mesoscopic fluid packets.

\subsection{The Continuum Boltzmann Equation}

In a continuum description, the single-particle distribution function $f_{1}(\mathbf{r}, \mathbf{v}, t)$ represents the density of fluid particles with position $\mathbf{r}$ and velocity $\mathbf{v}$ at time $t$, such that the density and velocity of the macroscopically observable fluid are given by $\rho(\mathbf{r}, t)=\int f_{1}(\mathbf{r}, \mathbf{v}, t) \mathrm{d} \mathbf{v}$ and $\mathbf{u}(\mathbf{r}, t)=\int f_{1}(\mathbf{r}, \mathbf{v}, t) \mathbf{v d} \mathbf{v}$ respectively. In the non-interacting, long mean free path limit, with no externally applied forces, the evolution of this function is described by the famous Boltzmann equation,

$$
\left(\partial_{t}+\mathbf{v} \cdot \nabla\right) f_{1}=\Omega\left[f_{1}\right] .
$$

The left hand side of the equation describes changes in the distribution function due to free particle motion; the right hand side contains the collision operator $\Omega$, describing changes due to pairwise collisions. Typically, this is an integral expression which can be hard to work with, so it is often simplified [28] to the linear Bhatnagar-Gross-Krook, or BGK form:

$$
\Omega[f] \simeq-\frac{1}{\tau}\left[f-f^{(\mathrm{eq})}\right]
$$

The BGK collision operator describes the relaxation, at a rate controlled by a characteristic time $\tau$, towards a Maxwell-Boltzmann equilibrium distribution $f^{(\mathrm{eq})}$. While this is a drastic simplification, it can be shown that distributions governed by the Boltzmann-BGK equation conserve mass, momentum, and energy, and obey a non-equilibrium form of the Second Law of Thermodynamics [29]. Moreover, it can be shown [30, 29] that the wellknown Navier-Stokes equations for macroscopic fluid flow are obeyed on coarse length and time scales by such distributions. 


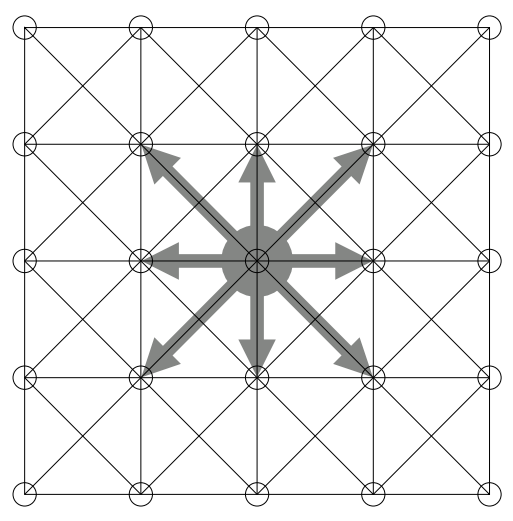

Figure 1: A lattice on which the Boltzmann equation may be discretized. Particles are only permitted to occupy positions shown by circles. Particles occupying the central point are permitted to be either at rest, or to have one of the eight possible discrete velocities $\mathbf{c}_{i}$ indicated by the grey arrows.

\subsection{The Lattice Boltzmann Equation}

In a lattice Boltzmann formulation, the single-particle distribution function is discretized in time and space. The positions $\mathbf{r}$ on which $f_{1}(\mathbf{r}, \mathbf{v}, t)$ is defined are restricted to points $\mathbf{r}_{i}$ on a lattice, and the velocities $\mathbf{v}$ are restricted to a set $\mathbf{c}_{i}$ joining points on the lattice; hence, $f_{i}(\mathbf{r}, t)=f\left(\mathbf{r}, \mathbf{c}_{i}, t\right)$ represents the density of particles at lattice site $\mathbf{r}$ travelling with velocity $\mathbf{c}_{i}$, at timestep $t$. The density and velocity of the simulated fluid are now given by

$$
\begin{gathered}
\rho(\mathbf{r})=\sum_{i} f_{i}(\mathbf{r}) \\
\mathbf{u}(\mathbf{r})=\sum_{i} f_{i}(\mathbf{r}) \mathbf{c}_{i}
\end{gathered}
$$

The lattice must be chosen carefully [31] to ensure isotropic behaviour of the simulated fluid. It can be shown [32] that the lattice Boltzmann equation may be rigorously derived by discretizing the continuum Boltzmann equation; alternatively, it may be regarded as a Boltzmann-level approximation of its ancestor, the LGA [33].

The discretized Boltzmann-level description of the fluid may now be evolved according to a two-step procedure. In the collision step, particles at each lattice site are redistributed across the velocity vectors: this process corresponds to the action of the collision 
operator, and usually takes the BGK form:

$$
f_{i} \leftarrow f_{i}-\frac{1}{\tau}\left[f_{i}-N_{i}\right]
$$

where $N_{i}=N_{i}(\rho(\mathbf{r}), \mathbf{u}(\mathbf{r}))$ is a polynomial function of the local density and velocity, which may be found by discretizing the well-known Maxwell-Boltzmann equilibrium distribution.

In the advection step, values of the post-collisional distribution function are propagated to adjacent lattice sites: this corresponds to particles streaming along their velocity vectors, and is the discretized equivalent of the left-hand side of the continuum Boltzmann equation:

$$
f_{i}\left(\mathbf{r}+\mathbf{c}_{i}\right) \leftarrow f_{i}(\mathbf{r})
$$

Overall, the system obeys the lattice Boltzmann equation (LBE), produced by combining the two evolution steps:

$$
f_{i}(\mathbf{r}, t+1)-f_{i}(\mathbf{r}, t)=\Omega[f]=-\frac{1}{\tau}\left[f_{i}(\mathbf{r}, t)-N_{i}(\rho, \mathbf{u})\right]
$$

It can be shown that the resulting macroscopic density and velocity fields obey the NavierStokes equations [34].

A well-known drawback of the lattice Boltzmann method is that it is typically not guaranteed to be numerically stable, and will crash or produce physically unreasonable results if, for example, the forcing rate applied to a fluid is too high or if the interparticle interaction strength is set too high.

\subsection{Multicomponent Interacting lattice Boltzmann Scheme}

There are several schemes for generalizing the lattice Boltzmann algorithm to treat multicomponent fluids, including analogies with LGA [5], imposition of free-energy functionals [35], discretization of a modified form of the continuum Boltzmann equation [36], or inclusion of an explicit forcing term in the collision operator. The lattice Boltzmann algorithms described in this paper use the last approach, due to Shan and Chen [37].

The single-particle distribution function $f_{i}$ may be extended to the form $f_{i}^{\sigma}$, where each component is denoted by a different value of the superscript $\sigma$, so that the density and momentum of a single component $\sigma$ are given by $\rho^{\sigma}=\sum_{i} f_{i}^{\sigma}$ and $\rho^{\sigma} \mathbf{u}^{\sigma}=\sum_{i} f_{i}^{\sigma} \mathbf{c}_{i}$ respectively. The lattice BGK equation (8) now takes the form

$$
f_{i}^{\sigma}(\mathbf{r}, t+1)-f_{i}^{\sigma}(\mathbf{r}, t)=-\frac{1}{\tau^{\sigma}}\left[f_{i}^{\sigma}-N_{i}\left(\rho^{\sigma}, \mathbf{v}^{\sigma}\right)\right]
$$


The velocity $\mathbf{v}^{\sigma}$ is found by calculating a weighted average velocity

$$
\mathbf{u}^{\prime}=\left(\sum_{\sigma} \frac{\rho^{\sigma}}{\tau^{\sigma}} \mathbf{u}^{\sigma}\right) /\left(\sum_{\sigma} \frac{\rho^{\sigma}}{\tau^{\sigma}}\right)
$$

and then adding a term to account for external forces,

$$
\mathbf{v}^{\sigma}=\mathbf{u}^{\prime}+\frac{\tau^{\sigma}}{\rho^{\sigma}} \mathbf{F}^{\sigma}
$$

The force term $\mathbf{F}^{\sigma}$ can take the form $g \rho^{\sigma} \hat{\mathbf{z}}$ to produce a gravitational force acting in the $z$-direction. In order to produce nearest-neighbour interactions between components, it assumes the form

$$
\mathbf{F}^{\sigma}=-\psi^{\sigma}(\mathbf{x}) \sum_{\bar{\sigma}} g_{\sigma \bar{\sigma}} \sum_{i} \psi^{\bar{\sigma}}\left(\mathbf{x}+\mathbf{c}_{i}\right) \mathbf{c}_{i},
$$

where $\psi^{\sigma}(\mathbf{x})=\psi^{\sigma}\left(\rho^{\sigma}(\mathbf{x})\right)$ is an effective charge for component $\sigma ; g_{\sigma \bar{\sigma}}$ is a coupling constant controlling the strength of the interaction between two components $\sigma$ and $\bar{\sigma}$. If $g_{\sigma \bar{\sigma}}$ is set to zero for $\sigma=\bar{\sigma}$, and a positive value for $\sigma \neq \bar{\sigma}$ then, in the interface between bulk regions of each component, particles experience a force in the direction away from the interface, producing immiscibility. In two-component systems, it is usually the case that $g_{\sigma \bar{\sigma}}=g_{\bar{\sigma} \sigma}=g_{b r}$.

Amongst other things, this model has been used to simulate spinodal decomposition [38, 39], polymer blends [40], liquid-gas phase transitions [41], and flow in porous media [42].

\subsection{Amphiphilic lattice Boltzmann}

As with many other mesoscale fluid methods, amphiphilic fluids may be treated in the LB framework by introducing a new species of particle with an orientational degree of freedom [43]. The particles of this species are each given a vector dipole moment $\mathbf{d}$ which has maximum magnitude $d_{0}$, corresponding to complete alignment of the constituent molecules. This is represented in the model by a dipole field $\mathbf{d}(\mathbf{x}, t)$ representing the average orientation of any amphiphile present at site $\mathbf{x}$. In the advection step, values of $\mathbf{d}(\mathbf{x}, t)$ are propagated around the lattice according to

$$
\rho^{\mathrm{s}}(\mathbf{x}, t+1) \mathbf{d}(\mathbf{x}, t+1)=\sum_{i} \tilde{f}_{i}^{\mathrm{s}}\left(\mathbf{x}-\mathbf{c}_{i}, t\right) \tilde{\mathbf{d}}\left(\mathbf{x}-\mathbf{c}_{i}, t\right)
$$


where tildes denote post-collision values. During the collision step itself, the dipole moments evolve in a BGK process controlled by a dipole relaxation time $\tau_{d}$ :

$$
\tilde{\mathbf{d}}(\mathbf{x}, t)=\mathbf{d}(\mathbf{x}, t)-\frac{1}{\tau_{d}}\left[\mathbf{d}(\mathbf{x}, t)-\mathbf{d}^{(\mathrm{eq})}(\mathbf{x}, t)\right]
$$

The equilibrium dipole moment $\mathbf{d}^{(\mathrm{eq})}$ is aligned with the colour field $\mathbf{h}$ :

$$
\mathbf{d}^{(\mathrm{eq})} \simeq \frac{\beta d_{0}}{3} \mathbf{h}
$$

The colour field contains a component $\mathbf{h}^{c}$ due to coloured particles such as oil and water, and a part $\mathbf{h}^{s}$ due to dipoles. The former can be found from the populations of surrounding lattice sites,

$$
\mathbf{h}^{c}=\sum_{\sigma} q^{\sigma} \sum_{i} \rho^{\sigma}\left(\mathbf{x}+\mathbf{c}_{i}\right) \mathbf{c}_{i}
$$

where $q^{\sigma}$ is a colour charge, such as +1 for red particles, -1 for blue particles, and 0 for amphiphile particles. The field due to other dipoles turns out to be given by

$$
\mathbf{h}^{s}(\mathbf{x}, t)=\sum_{i}\left[\sum_{j \neq 0} f_{i}^{s}\left(\mathbf{x}+\mathbf{c}_{i}, t\right) \theta_{j} \cdot \mathbf{d}_{i}\left(\mathbf{x}+\mathbf{c}_{j}, t\right)+f_{i}^{s}(\mathbf{x}, t) \mathbf{d}_{i}(\mathbf{x}, t)\right],
$$

where the second-rank tensor $\theta_{j}$ is defined in terms of the unit tensor $\mathbf{I}$ and lattice vector $\mathbf{c}_{j}$ as

$$
\theta_{j}=\mathbf{I}-\frac{D}{c^{2}} \mathbf{c}_{j} \mathbf{c}_{j}
$$

In the presence of an amphiphilic species, the force on an oil or water particle includes an additional term $\mathbf{F}^{\sigma, s}$ to account for the colour field due to the amphiphiles. By treating an amphiphilic particle as a pair of oil and water particles with a very small separation $\mathbf{d}$, and Taylor-expanding in $\mathbf{d}$, it can be shown that this term is given by

$$
\mathbf{F}^{\sigma, s}(\mathbf{x}, t)=-2 \psi^{\sigma}(\mathbf{x}, t) g_{\sigma s} \sum_{i \neq 0} \tilde{\mathbf{d}}\left(\mathbf{x}+\mathbf{c}_{i}, t\right) \cdot \theta_{i} \psi^{s}\left(\mathbf{x}+\mathbf{c}_{i}, t\right)
$$

where $g_{\sigma s}$ is a constant controlling the strength of the interaction between amphiphiles and non-amphiphiles.

While they do not possess a net colour charge, the amphiphiles also experience a force due to the colour field, consisting of a part $\mathbf{F}^{s, c}$ due to ordinary species, and a part $\mathbf{F}^{s, s}$ 
due to other amphiphiles. These terms are given by

$$
\begin{gathered}
\mathbf{F}^{s, c}=2 \psi^{s}(\mathbf{x}, t) \tilde{\mathbf{d}}(\mathbf{x}, t) \cdot \sum_{\sigma} g_{\sigma s} \sum_{i \neq 0} \theta_{i} \psi^{\sigma}\left(\mathbf{x}+\mathbf{c}_{i}, t\right) \\
\mathbf{F}^{s, s}=-\frac{4 D}{c^{2}} g_{s s} \psi^{s}(\mathbf{x}) \sum_{i}\left\{\tilde{\mathbf{d}}\left(\mathbf{x}+\mathbf{c}_{i}\right) \cdot \theta_{i} \cdot \tilde{\mathbf{d}}(\mathbf{x}) \mathbf{c}_{i}\right. \\
\left.+\left[\tilde{\mathbf{d}}\left(\mathbf{x}+\mathbf{c}_{i}\right) \tilde{\mathbf{d}}(\mathbf{x})+\tilde{\mathbf{d}}(\mathbf{x}) \tilde{\mathbf{d}}\left(\mathbf{x}+\mathbf{c}_{i}\right)\right] \cdot \mathbf{c}_{i}\right\} \psi^{s}\left(\mathbf{x}+\mathbf{c}_{i}\right) .
\end{gathered}
$$

To summarize, the interactions between fluid components are governed by the coupling constants $g_{b r}, g_{c s}$, and $g_{s s}$, controlling the interaction between different sorts of coloured particles, between coloured particles and amphiphiles, and between the amphiphiles.

While the form of the interactions seems straightforward at a mesoscopic level, it is essentially phenomenological, and it is not necessarily easy to relate the interaction scheme or its coupling constants to either microscopic molecular characteristics, or to macroscopic phase behaviour. Some theoretical progress has been made in relating LGA amphiphile models to an underlying microscopic model [44], although macroscopic behaviour is very sensitive not only to the values of the coupling constants, but to the concentrations of each species present, inter alia. Different values of these parameters will give rise to a wide variety of different phases [21], such as spherical and wormlike micelles, sponges, lamellae, or droplets: the phase behaviour can be very difficult to predict beforehand from the simulation parameters, and brute-force parameter searches are often resorted to [24].

\section{The practicalities of the lattice Boltzmann method}

If the sites of a lattice Boltzmann grid are evolved according to the algorithm described in Section 3, then the state of each site at a given timestep depends only on its state and the state of the neighbouring cells at the previous timestep, so that LB can be considered a form of cellular automaton [4, 3]. This spatial locality of the algorithm translates into memory locality in implementation, allowing for efficient performance on contemporary commodity computer architectures which use caching techniques to improve the speed of memory access, but also enables extremely efficient implementation on massively parallel computer architectures since, for a lattice split across CPUs (spatial domain decomposition), only the state of the lattice sites at the edge of each CPU's chunk of the lattice must 
be communicated to other CPUs. A more detailed examination of LB performance and a comparison with LGA is available in [45].

The following sections describe how two existing lattice Boltzmann codes were modified to allow for different forms of computational steering. One code, LB2D, is a lightweight, single-CPU solver for two-dimensional problems; steering was not directly bolted on to this code, but instead a high-level scripting interface was added to allow simulations to be controlled at runtime, either through high-level scripts, interactive manipulation, or other processes. The other code, LB3D, is a solver for three-dimensional problems involving ternary amphiphilic fluids, designed for use on distributed-memory parallel processing architectures; steering was added by interfacing the code to a separate steering library. ${ }^{1}$

\subsection{Design of a typical lattice Boltzmann code}

The lattice Boltzmann codes we examine in this article each revolve around a single data structure, which encapsulates the entire state of a simulation at a single instant in time. Specifically, this data structure contains the complete state of the lattice, with the value of $f_{i}^{\sigma}(\mathbf{x})$ for all values of $\sigma, i$, and $\mathbf{x}$; and also a set of simulation parameters. These parameters may be divided into two categories: parameters which are static and unchanging, such as the dimensions of the simulation lattice or parameters describing the initial state of the system, and parameters which could conceivably be changed during the course of the simulation, such as coupling constants and forcing rates. The code is then structured as a set of methods which act upon the data in this structure.

These methods can be loosely grouped into categories. 'Constructor' and 'destructor' methods allocate new simulation objects and free the memory associated with old ones; initialization methods initialize the state of a simulation object before commencing a given simulation run. IO methods write simulation data to disk, and also modify simulation data according to data saved on disk. These methods can load and save complete simulation states to and from disk, as well as loading, for example, porous media data, and saving information such as the fluid density field to disk. The save-data methods consist of two parts: one generic routine which produces a block of data corresponding to a physical field (such as density or pressure), and another routine which saves this block of data to disk in a specific format (such as raw binary, a portable binary format known as $\mathrm{XDR}$ [46], or a portable image format known as PNG).

Evolution methods perform advection or collision processes on a simulation object, and generally consume the majority of the CPU time in a given simulation run. Finally,

\footnotetext{
${ }^{1}$ LB3D was recently awarded the gold-star rating for its excellent scaling properties with large models running on 1024 processors on HPCx, the UK's fastest supercomputer.
} 
boundary condition methods alter the lattice, for example, to maintain a constant fluid density or composition at the edges of the simulated region.

\subsection{Traditional simulation methodology and its drawbacks}

Traditionally, large, compute-intensive simulations are run non-interactively. A text file describing the initial conditions and parameters for the course of a simulation is prepared, and then the simulation is submitted to a batch queue, to wait until there are enough resources available to run the simulation. The simulation runs entirely according to the prepared input file, and outputs the results to disk for the user to examine later.

This mode of working is sufficient for many simple investigations of mesoscale fluid behaviour; however, it has several drawbacks. Firstly, consider the situation where one wishes to examine the dynamics of the separation of two immiscible fluids: this is a subject which has been of considerable interest in the modelling community in recent years [39, 47]. Typically, a guess is made as to how long the simulation must run before producing a phase separation, and then the code is run for a fixed number of timesteps. If a phase transition does not occur within this number of timesteps, then the job must be resubmitted to the batch queue, and restarted. However, if a phase transition occurs in the early stages of the simulation, then the rest of the compute time will be spent simulating an equilibrium system of very little interest; worse, if the initial parameters of the system turn out not to produce a phase separation, then all of the CPU time invested in the simulation will have gone to waste.

Secondly, the input file often takes the simple form of a list of parameters and their values, but this may not be sufficiently expressive to describe the boundary conditions one may wish to apply, or the conditions under which they are to be applied. For example, to simulate the flow of a fluid mixture through a porous medium, it is necessary to equilibrate the flow of a single component through the medium first, before introducing the fluid mixture, in order to prevent the behaviour of the mixture from being affected by transients present as the flow field develops.

\subsection{High-level control of simulation codes: scripting}

For every new and complicated boundary condition one wishes to impose, it is in principle possible to write a corresponding new subroutine in the simulation code, add an option in the input file to switch this boundary condition on or off, and recompile the simulation code. However, in practice this leads to redundancy and overcomplication, or "bloat", in the simulation code, and also to excessively complicated or verbose input files. Bloated code will, in the long term, become difficult to maintain or change, and more complicated input file syntax makes it harder for new users to learn how to use the code. 
An alternative strategy is to abandon the concept of an input file altogether, and instead to control the simulation from a script written in a high-level language. This has several advantages.

Firstly, provided that enough access to the simulation data structures is provided to the scripting layer, new boundary conditions may be formulated, tested, and run with ease, without requiring the code to be recompiled. The core of the number-crunching code stays small and maintainable as a result.

Secondly, a high-level language provides conditional and loop structures, so simulations may be given much more detailed instructions than simply to run for a fixed number of timesteps: for example, a simulation of fluid phase separation could be instructed to run until the fluid components have separated to a certain degree, and to then stop.

Thirdly, writing the core of the simulation code in a language like $\mathrm{C}$ or Fortran but controlling its behaviour through a higher-level language allows the programmer to easily interface the simulation code with other components (such as image generation libraries) via the high-level language, which avoids the necessity of dealing with tedious low-level details of interfacing to many third-party libraries. This strategy also avoids incurring the performance penalty that would result from writing the entire simulation code in a higher-level language.

The approach of making a piece of code such as the simulation solver available as a self-contained reusable object to some higher-level "glue" layer is often termed "componentization". In this case, the glue layer is the high-level language; in the more general case, it could be a Grid fabric layer such as Web Services, allowing interoperation across the network of components running on different machines.

The high-level language chosen to script LB2D was Perl, a powerful language popular, amongst other things, for its ability to interface with, or 'glue', external components, and also for the wide variety of freely-available Perl code which can be easily accessed from scripts written in the language [48]. Constructing a functional Perl interface to the simulation code required little more than writing a formal description of the $\mathrm{C}$ subroutines comprising the simulation code [49]; interfacing code to other popular scripting languages such as TCL, Python, or Ruby is typically just as easy.

\subsection{Parameter space exploration using high-level scripting facility}

For an algorithm which runs the risk of encountering numerical instabilities, it is desirable to know the regions of parameter space in which one can operate without expecting to encounter such problems: for example, when studying the behaviour of an interface between two fluid components, it is useful to know how high the surface tension can be set before numerical instabilities are introduced, resulting in a simulation crash.

A crude approach to map out this region is to guess the size and location of a region 


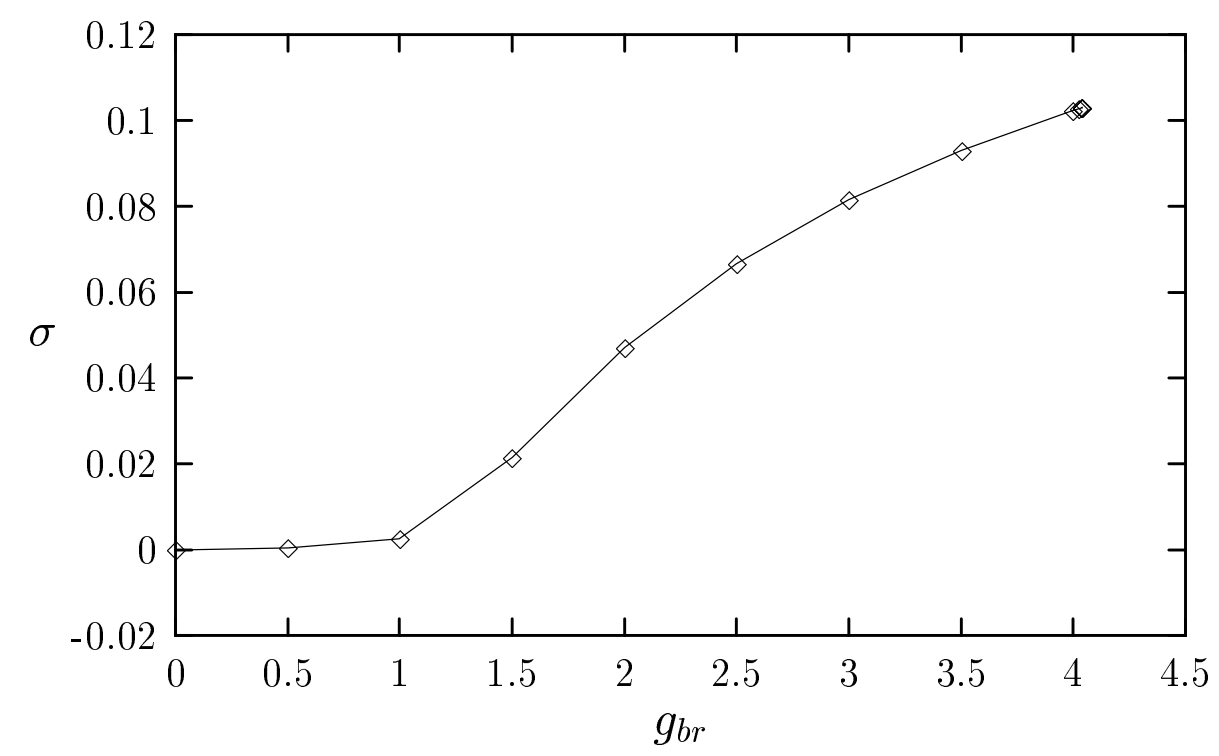

Figure 2: Graph of the surface tension $\sigma$ (in lattice units) of an interface between two immiscible fluids, against the value of the coupling constant $g_{b r}$. The high- $g_{b r}$ limit of the graph indicates the maximum value, beyond which simulations will become unstable.

of parameter space which will contain a region of stability, and blindly launch many simulations over this space.

A slightly more versatile approach made possible by scripting is to start with a knownstable point in parameter space, and an initial direction in parameter space. A simulation is started at the known-stable point, and if it completes successfully, another one is started at an adjacent point in parameter space, until eventually a numerically unstable regime is found; successive simulations can then be launched to home in on the location of the stable/unstable boundary.

In the Shan-Chen LB model, the interaction force between components, described in equation (12), gives rise to a surface tension at the interface between regions of different components; the strength of this interaction, and therefore the magnitude of the surface tension, is controlled by the coupling constant $g_{b r}$.

A simple parameter-space investigation is to take an interface between two immiscible fluids, and run simulations with increasing $g_{b r}$, and therefore increasing surface tension, until numerical instability sets in. The script controlling this process ensures that each simulation runs for long enough that the system reaches equilibrium. If a simulation succeeds, then the surface tension is raised; if not, then it is lowered, and the boundary is located using an interval bisection algorithm. The results of such an investigation are 


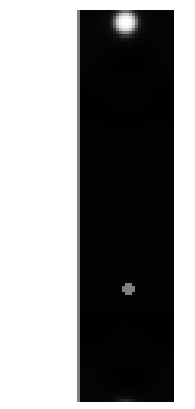

Timestep: 50

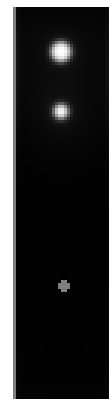

1000

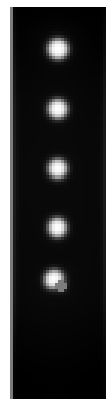

2650

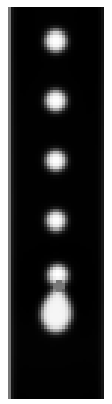

4450

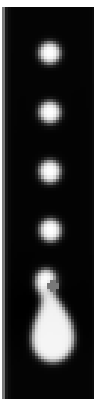

7250

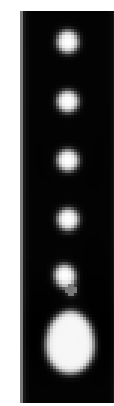

7850

Figure 3: Simulation of droplet coalescence on an obstacle during channel flow using scripted boundary conditions

shown in Figure 2, in which the surface tension was calculated for each value of $g_{b r}$ for which a simulation could successfully be run.

\subsection{Scripted boundary conditions}

The use of scripting allows extremely versatile and dynamic specification of boundary conditions in a simulation. Consider a stream of droplets flowing through a channel until they meet an obstacle: the droplets accumulate on the obstacle and coalesce to form a larger droplet, until the resulting droplet becomes too large and breaks free to travel further down the channel. Conventionally, one might investigate this situation by first running a simulation of single-component flow through the channel with the obstacle, until the velocity field equilibrates, and then starting a new simulation from this one in which droplets are added to the channel, either through manual intervention, or by writing an additional piece of code which periodically generates a new droplet near the entrance to the channel.

However, it is also possible to perform the simulation in one single run, using a script. This script equilibrates the single-component velocity field, and then automatically introduces a droplet to the channel entrance. It then waits, monitoring the simulation state, until the droplet has moved sufficiently far down the channel that a new one may be introduced without colliding with it. The results of such a simulation are shown in Figure 3 . the automatically-generated droplet stream is induced by the obstacle to coalesce into a single large droplet, which then breaks off shortly before timestep 7850. The advantage of this approach is that it does not require human intervention to restart the simulation after the velocity field has equilibrated, nor does it require a fixed droplet generation rate to be set: if such a rate were set slightly too high, then droplets may collide with one another 
before reaching the obstacle, a situation avoided by the use of a dynamically-specified boundary condition.

\section{Steering lattice-Boltzmann simulations through a generic library interface}

In this section, we present a discussion of the way in which we have implemented computational steering for LB3D within the ongoing RealityGrid project [50]. The RealityGrid project aims to enable the modelling and simulation of complex condensed matter structures at the molecular and mesoscale levels as well as the discovery of new materials using computational grids. The project also involves biomolecular applications and its long term ambition is to provide generic computational grid based technology for scientific, medical and commercial activities.

\subsection{Motivation}

Within RealityGrid, the way in which computational steering is implemented is driven by a desire to enable existing scientific computer programs (often written in Fortran90 and designed for multi-processor/parallel supercomputers) to be made steerable while minimising the amount of work required. Minimising the number of changes that a scientist must make to an existing program is important since it encourages him to take responsibility for this work. Consequently, the scientist understands the changes that are required and can continue to maintain the software in the future.

In the light of these requirements, we chose to implement the steering software as a library written in $\mathrm{C}$ and thus callable from a variety of languages (including $\mathrm{C}, \mathrm{C}++$ and Fortran90). The library completely insulates the application from any implementation details. For instance, the process by which messages are transferred between the steering client and the application (e.g. via files or sockets) is completely hidden from the application code.

Different scientists favour various techniques for writing programs intended to run on the specialist architectures of large supercomputers. Our steering library therefore does not assume or prescribe any particular parallel-programming paradigm (e.g. message passing or shared memory).

Obviously, a scientist does not want a failure in the steering system (such as a loss of connection to the application) to result in valuable computing time being lost. We have therefore designed the steering protocol so that, insofar as is possible, the steering is never made critical to the simulation process. The protocol enables a steering client to attach and detach from a running application without affecting its state. 
The scientist's ability to monitor the state of his simulation and use this to inform his steering decisions plays a key role in computational steering. While a steering client provides some information via the simulation's monitored parameters, a visualisation of some aspect of the simulation's state is often required. In our architecture this visualisation is created by a second software component.

\subsection{Requirements}

In order to make use of the steering library, an application must satisfy certain requirements. In particular, the application must have a logical structure such that there exists a point (which we term a breakpoint) within a control loop at which it is possible to carry out the following steering tasks:

i) emit a consistent representation of the state of the application's steerable and monitored parameters;

ii) accept a change to one or more steered parameters;

iii) emit a consistent representation (data sample) of part of the system being simulated (e.g. for visualisation);

iv) take a checkpoint or restart from an existing checkpoint.

While all of these things must, theoretically, be possible at the breakpoint, it is up to the scientist as to how many of them his application actually supports. For instance, enabling the application to restart from a checkpoint during execution might be a difficult task and therefore need only be attempted if the scientist particularly wants the functionality that that facility will bring.

\subsection{The steering library}

The steering library itself consists of two parts: an application side and a client side. The client side is intended to be used in the construction of a steering client. We have developed a generic steering client using C++ and Qt (a GUI toolkit) [51] which is capable of steering any application that has been 'steering enabled' using the library.

The steering library itself supports a variety of features. These include the facility for the application to register both monitored (read-only) and steerable (changed only through user interaction) parameters. Beyond this facility, the library supports a set of predefined commands such as 'pause', 'resume', 'detach' and 'stop.' In addition to these predefined commands, the library also allows the user to instruct the application to emit or 
consume any data sets that it has previously registered. Similarly, the user may instruct the application to take a checkpoint or restart from one which the application has registered.

The latter functionality is particularly important since it provides the basis of a system that allows the scientist to 'rewind' a simulation (by restarting from a previous checkpoint). Having done so, it can then be run again, perhaps after having steered some parameter or altered the frequency with which data from the simulation is recorded. The GRASPARC project [52] is an example of another system with this functionality.

In order to maximise the flexibility of the library, we use a system of 'reverse communication' with the application. This means that, for most actions, the library simply notifies the application of what the latter needs to do. It is then the application's responsibility to carry out the task, possibly using utility routines from the steering library. This is consistent with the philosophy mentioned earlier, of allowing the scientist to decide how much steering functionality he wishes to implement.

The steering library currently uses files for transmission of the steering messages. This means either that the application and the steering client must have access to the same disk or that some other software (known as 'middleware') takes responsibility for transferring the files between specific locations on the computers running the application and the client. Work in progress will lift this restriction by introducing direct communication between the application and client.

\subsection{Computational steering with LB3D}

As noted earlier, our parallel three-dimensional lattice-Boltzmann code (LB3D) has been interfaced to the RealityGrid steering library, which allows the user to steer all parameters of the simulation including coupling constants, fluid densities, relaxation times and even data dumping frequencies. Steerable data dumping frequencies enable the user to increase the amount of generated data for parts of the simulation where the effects of interest are happening. This helps to save an expensive resource, namely disk space.

In addition to the features the steering library provides, LB3D has its own logging and replay facilities which permit the user to 'replay' a steered simulation. This is an important feature since it allows the data from steered simulations to be reproduced without human intervention. Moreover, this feature can be used as an 'auto-steerer', i.e. multiple simulations which read different input-files at startup and are 'steered' in the same way can be launched without the need for human intervention during the simulation. One application of this particular feature is for studies of how changes in parameters affect a simulation that has evolved for a given number of timesteps. Another application is the automatic adaptation of data dumping or checkpointing frequencies. If the user has found from a manually steered simulation that no effects of interest are expected for a given number of initial timesteps, he can reduce the amount of data written to disk for early 
times of the simulation.

All steered LB3D simulations that are reported in this paper were performed on 64 processors of an SGI Origin 3800 in Manchester, UK. For data visualisation we used the Visualization Toolkit (VTK) [53] on a workstation in London. We chose to run the steering client on the same workstation.

\subsubsection{Spinodal decomposition}

As an example of a typical steered simulation with LB3D, let us consider the miscibility of a binary fluid mixture. We are interested in the behaviour of the system for different values of the coupling constant $g_{b r}$ which controls the strength of the interaction between both fluids, which we call 'blue' and 'red' here (see equation (12)). By interacting with a single ongoing simulation, we can change $g_{b r}$ 'on the fly' and immediately see how the fluid mixture behaves. Depending on the phenomena we are interested in, we can 'steer' the fluid into miscible or immiscible states. This technique can as well be utilised to find optimal values of $g_{b r}$ to study spinodal decomposition. Spinodal decomposition takes place if an incompressible binary fluid mixture is forced into thermodynamically unstable regions of its phase diagram, i.e. below its spinodal temperature. In this case, the mixture starts to phase separate into domains of the two fluids. This effect is important in various industries because phase separations in products like paints or cosmetics have to be controlled carefully and many researchers have studied spinodal decomposition in detail [39, 38, 54, 55, 56, 57, 47, 58, 59, 60, 61].

Figure 4 shows snapshots of volume rendered 'colour' fields (see section 2 and 3 for details). The colour field describes the net force of different fluid species on a given lattice site. A value of zero is obtained if forces caused by both fluid types cancel each other. This takes place at the interface between the fluids. A 'colour' field greater than zero corresponds to areas where the 'blue' fluid dominates while negative values correspond to a domination of 'red' particles respectively. In figures 4(a-f), areas of 'blue' dominance are rendered in blue and 'red' areas close to the interface are visualised in red. In the remaining snapshots, the colouring is utilized to visualise the diffusion of one fluid species into areas where the other species is dominant.

As initial condition for the simulation of the $64^{3}$ system, we chose a mixture, where for both species, each vector on each lattice site is assigned a random occupation number between zero and 0.7. Relaxation times and masses are set to unity and the initial value of $g_{b r}$ is 0.001 . This value is too low for phase separation to occur. Therefore, $g_{b r}$ is slowly raised to a value of 0.01 . Within a few thousand timesteps, both phases start to separate and after 11000 timesteps of the simulation, a clear structure in the fluid densities can be observed. It takes until timestep 24000 for the mixture to reach a fully separated state (figure 4 $\mathrm{a}-\mathrm{f}$ ). At timestep 25000 we start to reduce $g_{b r}$ again in order to force the 

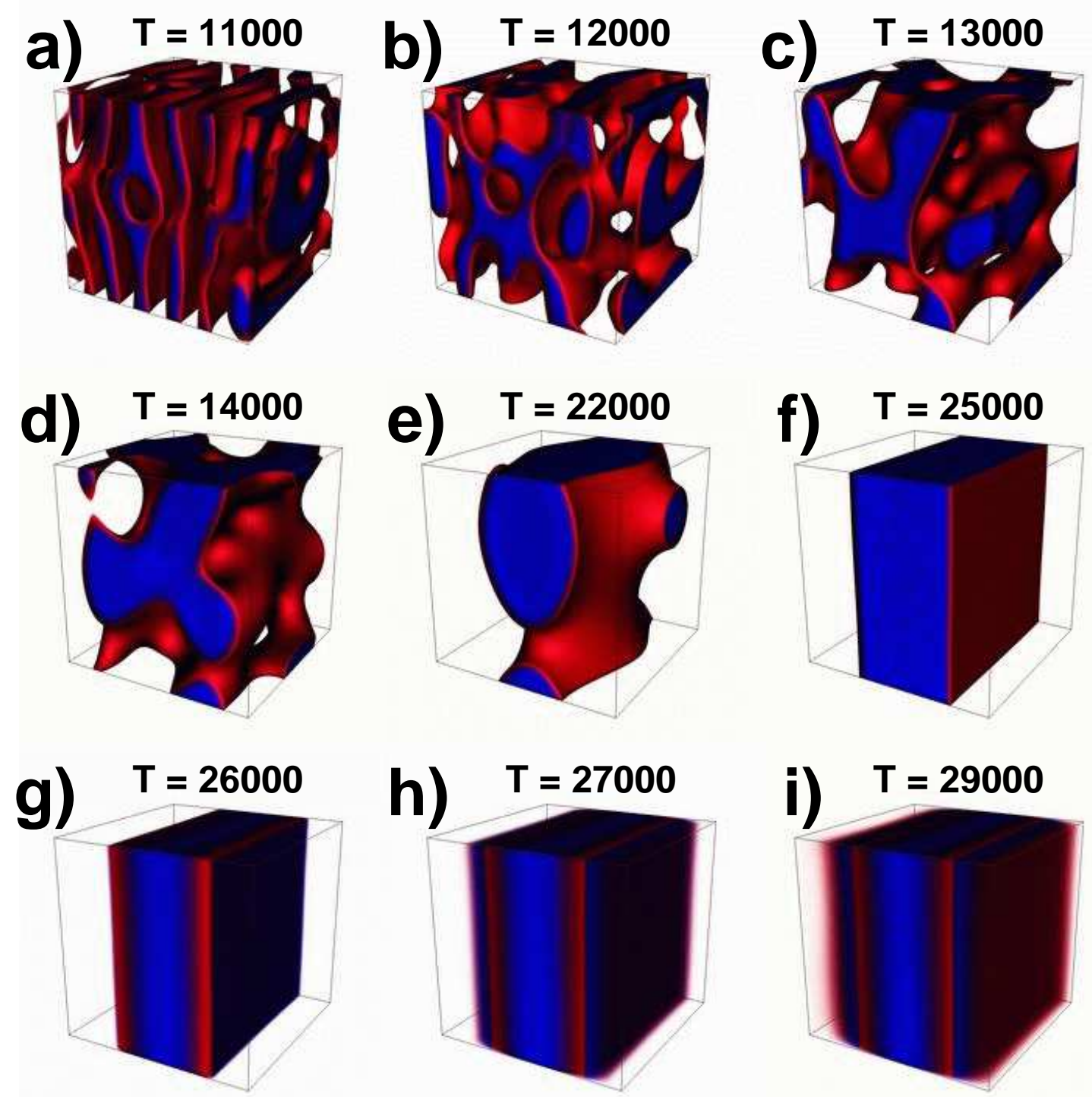

Figure 4: Snapshots of the 'colour' field from a steered lattice-Boltzmann simulation of a $64^{3}$ binary fluid mixture of red and blue particles using the LB3D code. The coupling constant $g_{b r}$ between both fluids is slowly raised from 0.001 to 0.01 during the inital phase of the simulation so that the phases start to separate until they reach a fully separated state after 25000 timesteps (a-f). Afterwards, the coupling is reduced to -0.001 so that the fluids become mixed again (g-i). In (a-f) the colour red is used to visualise where areas of 'red' dominance start and in (g-i) the colouring is utilized to show the diffusion of fluid particles into areas where the other species is dominant. 
fluids to mix again. The minimum value of the coupling strength used is -0.001 . Soon after reducing $g_{b r}$, red fluid particles start to diffuse into areas of blue dominance and vice versa. At timestep 29000 the system has arrived in a nearly fully mixed up state again (figures $4 \mathrm{~g}-\mathrm{i}$ ).

\subsubsection{Parameter searching}

The second example we give to demonstrate the usefulness of computational steering of three dimensional lattice-Boltzmann simulations is focused on parameter searches.

As noted previously, our simulations are very resource intensive. Single simulations might take between hours and days on a large number of processors of a parallel computer, and storing the generated data requires tens or hundreds of gigabytes of disk space. Typical system sizes of our simulations include $64^{3}, 128^{3}$ or $256^{3}$ lattices. The data written to disk for a single measuring timestep of a $256^{3}$ lattice requires about one gigabyte of disk space. For typical simulation lengths of 20000 timesteps and a measuring frequency of 100 timesteps, 200GB are needed. By reducing the lattice size to $64^{3}$, one is able to substantially reduce the amount of data to $3 \mathrm{~GB}$. However, due to the possible occurrence of finite size effects, such small lattice sizes are often not appropriate. Much of this data might turn out not to include physically interesting results or the data might be of limited use because the simulation parameters were not choosen correctly. Moreover, like other mesoscopic models, the lattice-Boltzmann method contains a number of free parameters (see section 3), resulting in high-dimensional parameter spaces, although only limited areas may be of interest. In addition, the phenomena of interest might occur within a limited time interval in the simulation only. In all these situations, very expensive compute resources are wasted.

The free parameters of our lattice-Boltzmann ternary amphiphilic fluid algorithm include the coupling constants between different fluid types (see section 3.3). The relationship between these parameters and experimentally available fluid properties is not well understood. Therefore, it is important to choose these parameters carefully so as to study a wide range of phenomena with one or a small number of parameter sets.

Traditionally, such 'optimal' parameter sets have been determined by 'task farming' approaches, that is by performing large numbers of small simulations concurrently, on a large parallel machine or on a large number of small individual machines [24]. This technique allows one at least in principle to 'scan' the entire parameter space. In practice, only subspaces can usually be investigated in detail, although these can be distributed. No human interaction is required after the jobs have been submitted, which makes it easy to use script based approaches for the generation of input files and job submission. However, the available computing resources are not used very efficiently. Not only is CPU time wasted in a task farm simulation, the amount of disk space needed to store the 
simulation data can be immense.

For example, we did large scale parameter searches for binary water-surfactant mixtures. The system size was $64^{3}$ and parameters studied were the surfactant-surfactant coupling constant $g_{s s}$, the surfactant-water coupling constant $g_{b s}$ (see equation (12)), and initial fluid densities. Masses and relaxation times were kept fixed at unity. We were only able to study small regions of the available parameter space, i.e. $g_{s s}$ was varied between -0.001 and -0.006 and values for $g_{b s}$ varied between -0.004 and -0.008 . The initial conditions were set as in section 5.4.1 but the maximum occupation numbers were varied from 0.2 to 0.7 for each fluid individually. In practice, we launched a number of simulations with different values for $g_{s s}, g_{b s}$ and initial densities and analysed the generated data afterwards. This analysis gave us an idea of interesting values for the parameters studied and, in principle, on the basis of these findings one could launch more simulations in order to investigate the system in more detail.

However, within a few wall-clock weeks of simulation time, we generated about 300GB of data and used about 30000 CPU hours on an SGI Origin 3800. While the simulations were performed in a highly automated manner within a couple of weeks, data analysis has been ongoing for months. Automation of the analysis of the generated data is much harder because it might be difficult to define the effects sufficiently well, or impossible to anticipate the effects in advance, or simply not worthwhile to invest additional effort in the development of algorithms to automate the process.

By considering this example, the disadvantage of conventional parameter searches is apparent: a significant fraction of the simulations performed in such a search employ parameters which do not admit interesting phenomena. Nonetheless, these simulations generate data that has to be analysed. In fact, it is not the elapsed computing time that makes parameter searches very time consuming, but extracting information from the data produced.

This analysis time can be significantly reduced by introducing human intuition into the simulation-analysis loop. The scientist doing the simulations and analysing the data is usually able to decide whether a parameter set is in a region of interest long before any given simulation finishes. By providing the scientist with the possibility to change simulation parameters on the fly, two goals can be achieved. First, he might be able to 'steer' the simulation into areas of interest: this improves the effective use of CPU cycles and reduces the amount of produced data. Second, analysing the reduced simulation output data is much less time consuming.

Figure 5]depicts a steered parameter search using the LB3D code. Instead of trying to cover the full parameter space, we only perform a limited number of steered simulations. We start with a random water-surfactant mixture with the surfactant-surfactant coupling constant $g_{s s}$ set to -0.003 and the water-surfactant coupling constant $g_{b s}$ set to -0.006 (figure 5 5 ). The lattice size is $64^{3}$ and initial maximum occupation numbers in this case 


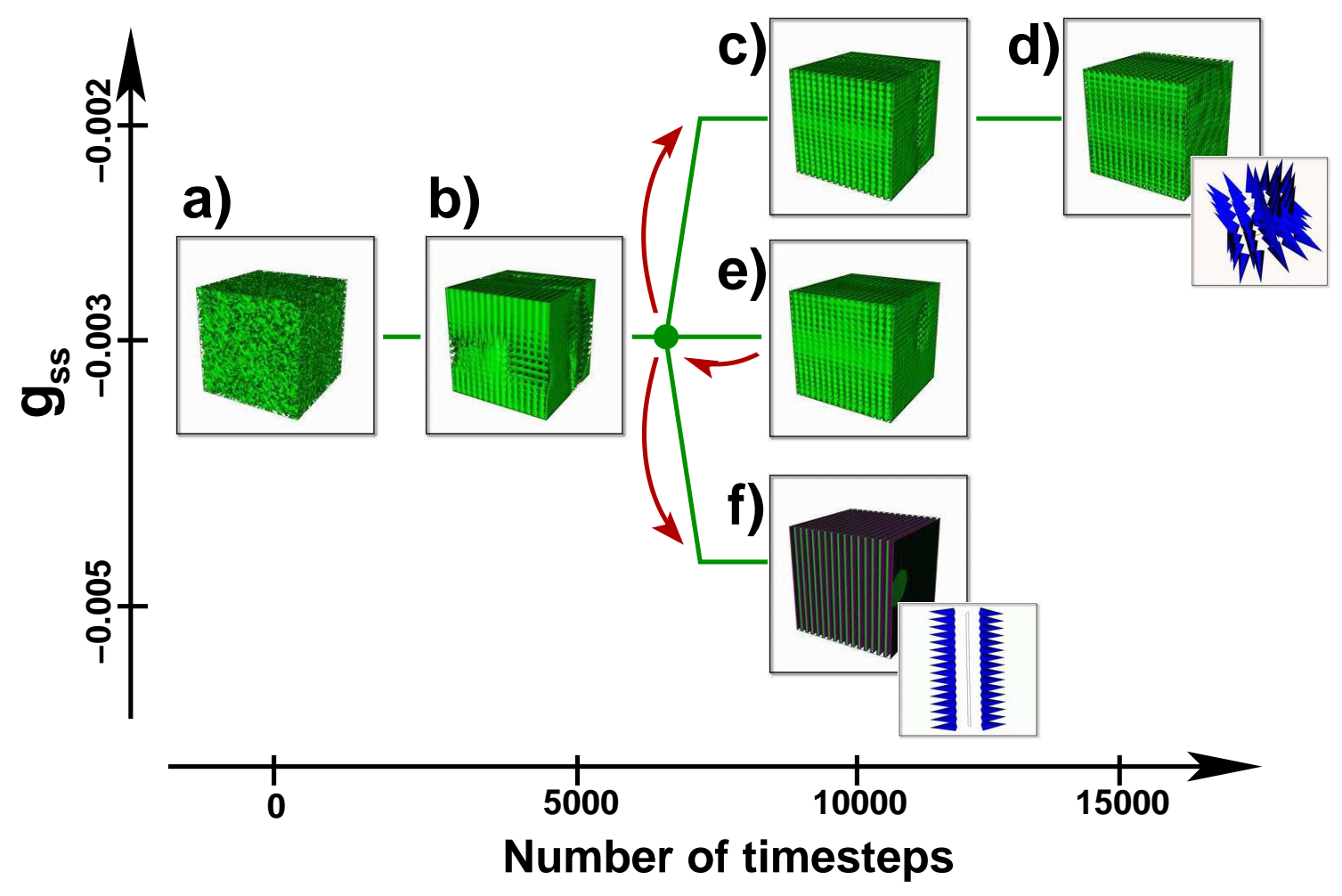

Figure 5: A steered parameter search is performed for a $64^{3}$ mixture of water and surfactant (see text for simulation details). The data visualised in the insets (a-f) is the volume rendered surfactant density, i.e. areas with densities higher than the average are coloured in green. We start at timestep zero with a random fluid mixture and $g_{s s}$ set to -0.003 (a). After 10000 timesteps we find a stable phase, i.e. a large fraction of the surfactant molecules forms spherical micelles (e). We 'rewind' the simulation to timestep 5000 (b) and change $g_{s s}$ to -0.002 . The system's state at timestep 10000 is only slightly different from (e), but still evolving in time. At timestep 15000 the micelles are much clearer than at timestep 10000, showing that a larger fraction of the surfactant molecules is involved in the micelle formation (d). The inset in (d) shows the orientations of surfactant particles around an area of low surfactant concentration: all of them point to regions of higher water concentration. We now rewind to timestep 5000 again and change $g_{s s}$ to -0.005 . At timestep 10000 the system has formed a lamellar structure (f), where the surfactant molecules are aligned in parallel bilayers between areas of high water density (see inset of (f)). 
are 0.7 for surfactant and 0.4 for water. The insets of figure 5 show volume rendered surfactant densities, where densities higher than the average are coloured in green. We monitor the state of the system while the simulation evolves and find a stable micellar phase after about 10000 timesteps (figure 5 b), i.e. a large fraction of surfactant molecules forms spherical micelles.

Since we are not able to detect any drastic changes for later timesteps, we 'rewind' the simulation back to timestep 5000 and change $g_{s s}$ to -0.002 . In this way we lower the interaction between surfactant molecules. Rewinding to an earlier simulation step is necessary because due to the low fluid velocities it takes a very long time in a system that is close to equilibrium for parameter changes to take effect. Steering the values of the coupling constants more drastically is not a solution to this problem because the simulation might become unstable and produce unphysical results or even crash.

Five thousand timesteps after 'rewinding', the system arrives in a state that is only slightly different from the $g_{s s}=-0.003$ case at timestep 10000. In order to investigate whether any more changes occur, we let the simulation evolve for a further 5000 timesteps and discover more well defined structures than before. A closeup of the dataset (see inset of figure 5 5 ) allows us to investigate the self-assembled spherical micelles in our system in more detail: the surfactant molecules visualised by the blue cones are pointing away from regions of low surfactant density, that is away from regions of high water density. The more well defined structure than in figure 5 r is due to a larger fraction of surfactant molecules being involved in forming micelles.

In order to investigate areas of the parameter space where the absolute value of $g_{s s}$ is higher, we 'rewind' the simulation again and change $g_{s s}$ to -0.005 . At timestep 10000, the system now finds itself in a drastically different state from the previous cases: water and surfactant form lamellae, the surfactant molecules align in parallel bilayers between areas filled with water. The colour purple is used here to visualise the interface between water and surfactant and the inset of figure 5: depicts how the surfactant molecules are aligned in this case.

Of course, a single steered simulation cannot by itself replace a full task farm parameter search, but a small number of steered simulations can provide a coarse grained overview of the available parameter space. By steering into areas of interest, one is able to dramatically reduce the resources required. Most of the analysis takes place during the simulation time itself and therefore required additional effort for offline analysis is reduced. Moreover, CPU time is further reduced because not every simulation has to start from timestep zero again. In the example given here, three conventional simulations of 15000 timesteps each sum up to 45000 simulation steps in total. In contrast, the steered simulations use only about 25000 timesteps because we do not have to rewind to timestep zero and can stop as soon as we cannot detect any further changes. Since the scientist interacts with the simulations, data dumping rates can be adapted during the run, thus 
reducing the requirements for disk storage even further.

\section{Computational steering}

As alluded to in previous sections, the problems associated with high performance computational science in general and large scale simulations in particular are not confined to merely finding resources with larger numbers of processors or memory. Simulations that require greater computational resources also require increasingly sophisticated and complex tools for the analysis and management of the output of the simulations. In Sections 4 and [5 we highlighted the limitations of a simple simulate-then-analyse approach and indicated how more sophisticated approaches help alleviate some of the problems. We then sketched some specific advantages of controlling the evolution of a computation, based upon the realtime analysis and visualisation of the status of a simulation, as opposed to the post facto analysis and visualisation of the output of a computation. The functionality that we refer to as computational steering enables the user to influence the otherwise sequential simulation and analysis phases by merging and interspacing them.

What additional requirements does computational steering place on computer systems? In order to computationally steer a simulation, one needs an interface to communicate with the simulation, which may be running on a remote machine. In addition to allowing parameters to be monitored and changed, this interface needs to offer the possibility of visualising complex data sets, for instance 3D isosurfacing and volume rendering. To enable intuitive interaction with a simulation, it is essential that visualisation can be perfomed sufficiently fast compared to changes taking place in the simulation. Visualisation of large and complex data sets typically requires high-end graphics hardware, which, like high-end computing resources, is not always available locally. Therefore, visualisation should be treated as a distributed resource as well, the need for which stems not only from computational steering but also from the requirements of high performance visualisation. The requirement to use more than one distributed resource simultaneously in turn raises more subtle issues associated with the requirements of sophisticated scheduling algorithms and techniques. Typical supercomputer centres currently make no provision for coallocation of resources, for example a compute node and a visualisation node. It would be desirable to be able to request resources for a computational steering session in advance and be assured of a certain quality of service during a session. Equally important is the requirement to be able to reserve substantial compute resources with small turn around time.

What advantages does computational steering provide the application scientist in return? We have described a few specific examples of how computational steering can increase a scientist's productivity. This increase in productivity is due to an increase in 
the throughput of hardware resources but equally important is the enhanced productivity due to a more effective computational science workflow bench (simulation-analysis loop) as a consequence of being able to use computational steering. There have been attempts to use computational steering as a novel approach to studying outstanding and important problems in biomolecular systems [62]. At the very least, computational steering complements existing techniques [63]. However, computational steering should not simply be thought of as an effective tool in the production and analysis phase of a simulation. It can provide the application scientist (often also the application developer) with greater flexibility in the development, debugging and validation phases of an application [8, 64, 65], where it complements rather than replaces other well established tools. Computational steering can also be extended to collaborative environments where several geographically distributed scientists can simultanously interact with one or more simulations from separate locations [66, 67, 68, 69, 70].

This sets the stage for a few remarks on what kind of computational science applications are suitable candidates for the use of computational steering. If an application requires barely a few seconds of computing time to simulate a physical process or effectively finish the simulation (say a fixed small number of iterations in a minimization routine), then the advantages of user intervention while the simulation is in progress are limited. Any overhead associated with interrupting such an inexpensive simulation will not be worth the gain bought by interactivity. At the other end of the spectrum, simulations that 'take forever' for any discernible changes to manifest as a consequence of user interaction are also not good candidates as the advantage from such interactivity is typically limited. A case in point are ab initio quantum mechanical molecular dynamics simulations, where even when only a small number of atoms are of interest, each step of the calculation may take several hours on a multiprocessor machine [71, 72]. Any changes initiated dynamically by the user for such simulations would take many hours to become manifest, clearly limiting their use. Thus it appears to be the case that simulations with a run time from several minutes to several hours (irrespective of the resources used), are ideally suited for interactive aspects of computational steering. It is important to distinguish between the role of steering in long running simulations as opposed to simulations with long response times to a pertubation. We have discussed the limited role of steering in the latter, but in the former case, steering can be useful for checking the progress of a long running simulation by connecting to the simulation, getting a sample and visualising it and then disconnecting after checking all is correct, thus enabling the scientist to use computational steering as 'simulation monitor' and as a safeguard against possible wastage of computational resources.

A few cautionary remarks are in place. Many physical systems have long equilibration time scales and suffer from finite size effects. In such cases, changing the parameters and taking the state immediately following the change to be the putative true state might be 
misleading. Moreover, many physical systems exhibit hysteresis, that is their properties at a given point in parameter space are dependent upon their history. Before computationally steering a simulation, it is imperative to determine if it displays hysteresis and, if so, how the use of steering may influence the analysis. Computational steering of diverse applications may involve different challenges, but in all cases an antidote to possible problems will be careful and consistent study rather than a refusal to adopt new analysis techniques.

We end this section by discussing why there has been comparatively limited acceptance or use of computational steering in scientific applications until now. If there is a lesson to be learnt from the evolution of computational science, it is that the complexity of doing something new and exciting has to be well hidden from the application scientist, i.e. it is essential to minimize the amount of implementation, learning, disruption and changes to the user interface, until the advantages of the new features are well established and very clearly seen to offset the cost of implementation. Ideally one would like, if possible, to just slip in the functionality where the user never knows or notices, but this may not be achievable in a computational steering context, when the application scientist often has to actually execute the functionality. Thus maximal effort will have to be invested into reducing the 'barrier of entry'. Until now, implementing computational steering has required a high degree of customization, but most scientists typically are not in a position to invest in the time-consuming task of developing the necessary tools; indeed, cooperation with specialists in visualisation and interfacing techniques has hitherto been vital. This problem is not helped by the fact that the requirements of a scientist might change during a project because new results from simulations lead future investigations in different directions than initially planned. This could result in the steering tools having to be adapted, which can be very costly [64, 65].

The question that logically follows is what can be done to address the relatively low acceptance of computational steering in computational science. We believe that most scientists are not cognisant of the advantages computational steering offers and thus unaware how they might benefit from steerable applications. Therefore, their simulations are done in the 'traditional' way, invoking long batch jobs and subsequently lengthy offline data analysis. In this paper we have outlined the advantages that computational steering has brought to our LB studies. Part of the purpose of this article is to encourage computational scientists to think about the enhanced ability and benefits that computational steering capabilities would bring to their scientific productivity, along the lines of our LB studies, but specific to their own applications. It is obvious that not all computational science problems are amenable to, or for that matter require, computational steering. However, we believe that documenting the advantages of computational steering in widely differing applications and areas will help bring greater acceptance of steering as a valid paradigm for computational science research. We also wish to emphasize that, as shown by the 
RealityGrid steering framework, by using the correct abstractions and good software engineering practices, implementing the required changes is much less effort than might otherwise be expected. Indeed many generic tools and libraries useful in program steering and data visualisation are now readily available $[8,73,7,74]$.

\section{Steering on Computational Grids: Current Status and Future Outlook}

Significant effort is being invested worldwide in Grid computing [75]. A basic premise of grid computing is to provide the infrastructure required to facilitate the collaborative sharing of resources. The grid aims to present the elements required for a computational task (e.g. calculation engine, filters, visualisation capability) as components which can be effectively and transparently coupled through the grid framework. In this scenario, any application or simulation code can be viewed simply as a data producing/consuming object on the grid and computational steering is a way of allowing a user to interact with such objects. As discussed in previous sections, a scientist using steering has heterogeneous and dynamic computational resource requirements, making the stated ability of the grid to collectively and transparently marshall diverse resources complementary to the primary motivation of computational steering. Thus a grid infrastructure that permits the coordination of heterogeneous and distributed computing resources provides a natural environment as well as a testbed for demonstrating the effectiveness of steering in computational science.

In our description of the RealityGrid steering framework in section [5, we did not mention the use of a computational grid or dependence on any underlying middleware requirement. This is because the RealityGrid steering framework is capable of being used on stand alone workstations as well as the most ambitious computational grids available in the world today. Equally important is the fact that our steering framework is not critically dependent on any one particular middleware although it does adhere to the best practises and the open standards currently being discussed within the Global Grid Forum (GGF) [75]. In the remainder of this section we will describe how the RealityGrid project uses the grid to implement computational steering.

In the same way that a high-fidelity simulation of a physical system often requires a supercomputer, so the visualisation of the (potentially) large data sets that these simulations produce also requires specialist hardware that few scientists have direct access to. Consequently, the visualisation component of the RealityGrid steering may well be on a machine other than the one the scientist is sitting in front of. This is consistent with our earlier proposal that visualisation be treated like a distributed resource. This then requires that the output (images) of the visualisation component be returned to the user's worksta- 
tion quickly enough to allow for full interactivity (e.g. to rotate, zoom, etc.). We currently use SGI's OpenGL VizServer software to perform this task; it takes the images directly from the rendering hardware on the visualisation machine, compresses them, transports them to the user's machine, decompresses and displays them [76]. This allows the scientist to interact with a remote visualisation, even over network links with relatively low bandwidth.

The outline traced above has been the basis for several successful computational steering demonstrations that we have performed within the past year. In our first demonstration at the UK e-Science All Hands Conference in Sheffield in September 2002, we used this technique to interact with a visualisation produced on an SGI Onyx300 in Manchester from a laptop in Sheffield with the computation performed in London. For this demonstration and the subsequent two, we used Unicore [77] as the underlying middleware to manage the file transfer aspects of the demonstration. At Supercomputing 2002, we used a trans-Atlantic link to interact with a visualisation on the Onyx300 in Manchester from a laptop in Baltimore, USA, the computation being performed on the SGI Origin 3800 in Manchester. In February of 2003 at the SGI VizSummit, we used a laptop in Paris to interact with simulations on 128 processors of the Origin 3800 in Manchester, visualising and steering being performed locally using the Onyx 300 facilities provided on the demonstration floor. Thus we have in the process performed computational steering using three different albeit transient grid scenarios: within the UK, trans-Atlantic and UK-continental Europe.

The grids used in these demonstrations were assembled especially for each event. However, the UK e-Science community has constructed an ambitious Level 2 Grid [78] that aims to provide the user community with a persistent grid. We have already deployed a preliminary RealityGrid LB3D application involving computational steering, using Globus (as opposed to Unicore, confirming the flexibility of our steering framework) on this Level 2 Grid, thus being amongst the first groups in the world to use a persistent grid for routine science requiring high performance computing and computational steering.

At the time of writing, the RealityGrid steering library supports both file-based and streaming (based on globus_io from the Globus project [79]) data transfer between the application and visualisation components. Communications between the application and the client is currently implemented by exchanging XML documents through a shared file system (XML is a widely accepted language specifying the syntax to mark-up data in computer documents).

We are in the process of implementing a more flexible architecture, based on the Open Grid Services Infrastructure [80]. As shown in Figure 6 communications between the application and client are routed through an intermediate steering grid service (SGS). The SGS provides the public interface through which clients can steer the application. In our 


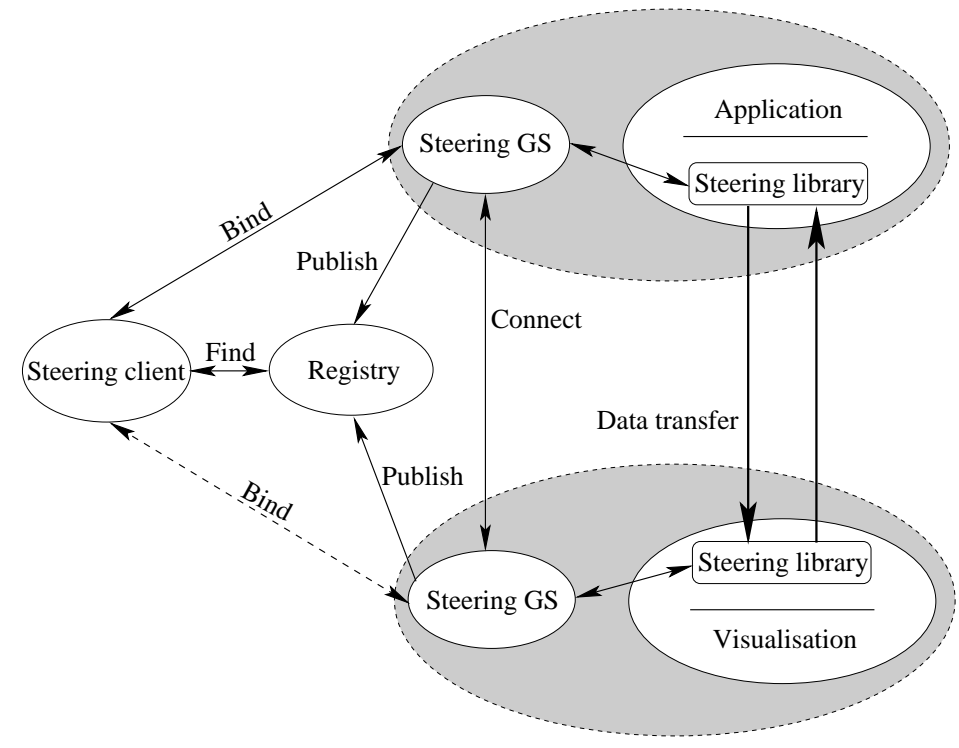

Figure 6: Architecture for RealityGrid steering within the Open Grid Services Infrastructure (OGSI). The application and client communicate by exchanging messages through intermediate grid services. The grid service (GS) provides the public interface through which clients can steer the applications.

architecture, the visualisation and application components appear on equal footing, and a visualisation can possess its own SGS. Each SGS publishes information about itself in a registry, which is used by clients to discover and bind to running applications, and can also be instrumental in bootstrapping the communications between the application and visualisation components. We note that the approach of exposing steering controls as grid services in a standard way could bring profound benefits in the form of interoperability between different implementations of computational steering.

\section{Conclusions}

This paper has described the work we have done to incorporate computational steering in mesoscale lattice Boltzmann simulations of binary and ternary immiscible and amphiphillic fluids. The scale and efficiency of these studies is set to increase dramatically with the advent of computational grids which are now becoming widely available within the UK, Europe, the USA and the Pacific Rim. 


\section{Acknowledgements}

We are grateful to ESPRC for funding much of this research through RealityGrid grant GR/R67699 and for providing access to SGI Origin 3800, Origin 2000, and CRAY T3E supercomputers at Computer Services for Academic Research (CSAR), Manchester, UK. We would also like to thank the University of Manchester for access to their SGI Onyx300 and HEFCE for funding the 16 processor dual pipe SGI Onyx2 at University College London. Jonathan Chin acknowledges Huntsman and Queen Mary, University of London, for funding his Ph.D. studentship. Jens Harting wishes to thank the European Commission Access to Research Infrastructures action of the 'Improving Human Potential Programme' for supporting his stay at Italy's national supercomputer centre in Bologna (CINECA) and the use of their local IBM SP4, SGI Origin 3800 and SGI Onyx 2 facilities.

\section{References}

[1] L. E. Reichl. A Modern Course in Statistical Physics. J. Wiley and Sons, second edition, 1998.

[2] J.-P. Rivet and J. P. Boon. Lattice Gas Hydrodynamics. Cambridge University Press, 2001.

[3] S. Succi. The Lattice Boltzmann Equation for Fluid Dynamics and Beyond. Oxford University Press, 2001.

[4] D. H. Rothman and S. Zaleski. Lattice Gas Cellular Automata. Cambridge University Press, 1997.

[5] A. K. Gunstensen, D. H. Rothman, S. Zaleski, and G. Zanetti. Lattice Boltzmann model of immiscible fluids. Phys. Rev. A, 43(8):4320-4327, 1991.

[6] W. Gu, J. Vetter, and K. Schwan. An annotated bibliography of interactive program steering. ACM SIG-PLAN Notices, 29(9):140-148, 1994.

[7] J. Prins, J. Hermans, G. Mann, L. Nyland, and M. Simons. A virtual environment for steered molecular dynamics. Future Generation Computer Systems, 15:485-495, 1999.

[8] W. Gu, G. Eisenhauer, and J. V. Karsten Schwan. Falcon: On-line monitoring and steering of parallel programs. Technical report, College of Computing, Georgia Institute of Technology, 1995. http://www.cc.gatech.edu/systems/projects/FALCON/. 
[9] L. E. Scriven and C. V. Sternling. The Marangoni effects. Nature, 187:186 - 188, 1960.

[10] J. B. Grotberg and D. P. G. III. A synopsis of surfactant spreading research. J. Colloid Interface Sci., 178:377-378, 1996.

[11] B. M. Boghosian and P. V. Coveney. A particulate basis for an immiscible lattice-gas model. Comp. Phys. Comm., 129(1-3):46-55, 2000.

[12] E. G. Flekkøy, P. V. Coveney, and G. D. Fabritiis. Foundations of dissipative particle dynamics. Phys. Rev. E, 62(2):2140-2157, 2000.

[13] P. J. Hoogerbrugge and J. M. V. A. Koelman. Simulating microscopic hydrodynamic phenomena with dissipative particle dynamics. Europhys. Lett., 19(3):155160, 1992.

[14] P. Español and P. Warren. Statistical mechanics of dissipative particle dynamics. Europhys. Lett., 30(4):191-196, 1995.

[15] P. V. Coveney and P. Español. Dissipative particle dynamics for interacting multicomponent systems. J. Phys. A: Math. Gen., 30:779-784, 1997.

[16] U. Frisch, B. Hasslacher, and Y. Pomeau. Lattice-gas automata for the Navier-Stokes equation. Phys. Rev. Lett., 56(14):1505-1508, 1986.

[17] S. Wolfram. Cellular automaton fluids 1: Basic theory. J. Stat. Phys., 45(3/4):471$526,1986$.

[18] U. Frisch, D. d'Humières, B. Hasslacher, P. Lallemand, Y. Pomeau, and J.-P. Rivet. Lattice gas hydrodynamics in two and three dimensions. Complex Systems, 1:649707, 1987.

[19] D. H. Rothman and J. M. Keller. Immiscible cellular-automaton fluids. J. Stat. Phys., 52(3-4):1119-1127, 1988.

[20] P. V. Coveney, J. Maillet, J. L. Wilson, P. W. Fowler, O. Al-Mushadani, and B. M. Boghosian. Lattice-gas simulations of ternary amphiphilic fluid flow in porous media. Int. J. Mod. Phys. C, 9(8):1479-1490, 1998.

[21] G. Gompper and M. Schick. Self-assembling amphiphilic systems. In C. Domb and J. Lebowitz, editors, Phase Transitions and Critical Phenomena, volume 16, pp 1-176. Academic Press, 1994. 
[22] P. J. Love, J. Maillet, and P. V. Coveney. Three-dimensional hydrodynamic latticegas simulations of binary immiscible and ternary amphiphilic flow through porous media. Phys. Rev. E, 64(061302), 2001.

[23] B. M. Boghosian, P. V. Coveney, and A. N. Emerton. A lattice-gas model of microemulsions. Proc. R. Soc. Lond. A, 452:1221-1250, 1996.

[24] B. M. Boghosian, P. V. Coveney, and P. J. Love. A three dimensional lattice-gas model for amphiphilic fluid dynamics. Proc. R. Soc. Lond. A, 456:1431, 2000.

[25] A. Malevanets and R. Kapral. Continuous-velocity lattice-gas model for fluid flow. Europhys. Lett., 44(5):552-558, 1998.

[26] Y. Hashimoto, Y. Chen, and H. Ohashi. Immiscible real-coded lattice gas. Comp. Phys. Comm., 129(1-3):56-62, 2000.

[27] T. Sakai, Y. Chen, and H. Ohashi. Formation of micelle in the real-coded lattice gas. Comp. Phys. Comm., 129(1-3):75-81, 2000.

[28] P. L. Bhatnagar, E. P. Gross, and M. Krook. Model for collision processes in gases. I. Small amplitude processes in charged and neutral one-component systems. Phys. Rev., 94(3):511-525, 1954.

[29] R. L. Liboff. Kinetic Theory: Classical, Quantum, and Relativistic Descriptions. Prentice-Hall, 1990.

[30] S. Chapman and T. G. Cowling. The Mathematical Theory of Non-uniform Gases. Cambridge University Press, second edition, 1952.

[31] Y. H. Qian, D. d'Humières, and P. Lallemand. Lattice BGK models for NavierStokes equation. Europhys. Lett., 17(6):479-484, 1992.

[32] X. He and L.-S. Luo. Theory of the lattice Boltzmann method: From the Boltzmann equation to the lattice Boltzmann equation. Phys. Rev. E, 56(6):6811-6817, 1997.

[33] G. R. McNamara and G. Zanetti. Use of the Boltzmann equation to simulate latticegas automata. Phys. Rev. Lett., 61(20):2332-2335, 1988.

[34] H. Chen, S. Chen, and W. H. Matthaeus. Recovery of the Navier-Stokes equations using a lattice-gas Boltzmann method. Phys. Rev. A, 45(8):5339-5341, 1992.

[35] M. R. Swift, E. Orlandini, W. R. Osborn, and J. M. Yeomans. Lattice-Boltzmann simulations of liquid-gas and binary fluid mixtures. Phys. Rev. E, 54(5):5041-5052, 1996. 
[36] L.-S. Luo. Theory of the lattice Boltzmann method: Lattice Boltzmann models for nonideal gases. Phys. Rev. E, 62(4):4982-4995, 2000.

[37] X. Shan and H. Chen. Lattice Boltzmann model for simulating flows with multiple phases and components. Phys. Rev. E, 47(3):1815-1819, 1993.

[38] J. Chin and P. V. Coveney. Lattice Boltzmann study of spinodal decomposition in two dimensions. Phys. Rev. E, 66(016303), 2002.

[39] N. González-Segredo, M. Nekovee, and P. V. Coveney. Three-dimensional latticeBoltzmann simulations of critical spinodal decomposition in binary immiscible fluids. Phys. Rev. E, in print. Available as e-print cond-mat/0301046 in Los Alamos Preprint Archive - http://www.arXiv.org/ .

[40] N. S. Martys and J. F. Douglas. Critical properties and phase separation in lattice Boltzmann fluid mixtures. Phys. Rev. E, 63:031205, 2001.

[41] X. Shan and H. Chen. Simulation of nonideal gases and liquid-gas phase transitions by the lattice Boltzmann equation. Phys. Rev. E, 49(4):2941-2948, 1994.

[42] N. S. Martys and H. Chen. Simulation of multicomponent fluids in complex threedimensional geometries by the lattice Boltzmann method. Phys. Rev. E, 53(1):743$750,1996$.

[43] H. Chen, B. M. Boghosian, P. V. Coveney, and M. Nekovee. A ternary lattice Boltzmann model for amphiphilic fluids. Proc. R. Soc. Lond. A, 456:2043-2047, 2000.

[44] P. J. Love. A particulate basis for a lattice-gas model of amphiphilic fluids. Phil. Trans. R. Soc. Lond. A, 360:345, 2002.

[45] P. J. Love, M. Nekovee, P. V. Coveney, J. Chin, N. González-Segredo, and J. M. R. Martin. Simulations of amphiphilic fluids using mesoscale lattice-Boltzmann and lattice-gas methods. Comp. Phys. Comm., in press. Available as e-print condmat/0212148 in Los Alamos Preprint Archive - http://www.arXiv.org/ .

[46] R. Srinivasan. XDR: External data representation standard. Network working group request for comments: 1832, Internet Engineering Task Force, 1995. http://www.ietf.org/rfc/rfc1832.txt.

[47] V. M. Kendon, M. E. Cates, I. Pagonabarraga, J. C. Desplat, and P. Bladon. Inertial effects in three-dimensional spinodal decomposition of a symmetric binary fluid mixture: A lattice Boltzmann study. J. Fluid Mech., 440:147-203, 2001. 
[48] The Comprehensive Perl Archive Network. http://www.cpan.org/ .

[49] T. Jenness and S. Cozens. Extending and Embedding Perl. Manning Publications, 2002.

[50] The RealityGrid project: http://www.realitygrid.org.

[51] Trolltech Qt - A GUI Toolkit, http://www.trolltech.com.

[52] K. W. Brodlie, L. A. Brankin, G. A. Banecki, A. Gay, A. Poon, and H. Wright. GRASPARC: A problem solving environment integrating computation and visualization. In G. M. Nielson and D. Bergeron, editors, Proceedings of IEEE Visualization 93 Conference, p 102. IEEE Computer Society Press, 1993.

[53] W. Schroeder, K. Martin, and B. Lorensen. The Visualization Toolkit: An Object Oriented Approach to 3D Graphics. Kitware, Inc., 3rd edition, 2003.

[54] P. V. Coveney and K. E. Novik. Computer simulations of domain growth and phase separation in two-dimensional binary immiscible fluids using dissipative particle dynamics. Phys. Rev. E, 54(5):5134-5141, 1996.

[55] F. J. Alexander, S. Chen, and D. W. Grunau. Hydrodynamic spinodal decomposition: Growth kinetics and scaling functions. Phys. Rev. B, 48(1):634-637, 1993.

[56] M. Grant and K. R. Elder. Spinodal decomposition in fluids. Phys. Rev. Lett., 82(1):14-16, 1999.

[57] H. Furukawa. Spinodal decomposition of two-dimensional fluid mixtures: A spectral analysis of droplet growth. Phys. Rev. E, 61(2):1423-1431, 2000.

[58] F. Perrot, C. K. Chan, and D. Beysens. Spinodal decomposition under shear: Towards a two-dimensional growth? Europhys. Lett., 9(1):65-70, 1989.

[59] R. B. Rybka, M. Cieplak, and D. Salin. Boltzmann cellular automata studies of the spinodal decomposition. Physica A, 222:105-118, 1995.

[60] F. J. Solis and M. O. de la Cruz. Hydrodynamic coarsening of binary fluids. Phys. Rev. Lett., 84(15):3350-3353, 2000.

[61] E. D. Siggia. Late stages of spinodal decomposition in binary mixtures. Phys. Rev. A, 20(2):595-605, 1979. 
[62] S. Izrailev, S. Sergey, B. Isralewitz, D. Kosztin, H. Lu, F. Molnar, W. Wriggers, and K. Schulten. Computational molecular dynamics: Challenges, methods, ideas. In P. Deuflhard et al., editors, volume 4 of Lecture Notes in Computational Science and Engineering, pp 39-65. Springer-Verlag, Berlin, 1998.

[63] I. Barry, M. Gao, and K. Schulten. Steered molecular dynamics and mechanical functions of proteins. Current Opinion in Structural Biology, 11:224-230, 2001.

[64] R. van Liere, J. Mulder, and J. van Wijk. Computational steering. Future Generation Computer Systems, 12(5):441-450, 1997.

[65] J. van Wijk, R. van Liere, and J. Mulder. Bringing computational steering to the user. In H. Hagen, G. Nielson, and F. Post, editors, Scientific visualization, pp 304-313. IEEE Computer Society, 2000.

[66] I. Foster and C. Kesselman. Computational grids. In I. Foster and C. Kesselman, editors, The Grid: Blueprint for a New Computing Infrastructure, pp 15-25. Morgan Kaufmann, 1999.

[67] T. DeFanti and R. Stevens. Teleimmersion. In I. Foster and C. Kesselman, editors, The Grid: Blueprint for a New Computing Infrastructure, pp 131-155. Morgan Kaufmann, 1999.

[68] K. Brodlie, S. Mason, M. Thompson, M. Walkley, and J. Wood. Reacting to a crisis: benefits of collaborative visualization and computational steering in a grid environment. In paper presented at the UK e-Science All Hands Conference, Sheffield, 2002.

[69] M. Walkley, J. Wood, and K. Brodlie. A Distributed Co-operative Problem Solving Environment. Computational Science - ICCS 2002, LNCS 2329, Springer, 2002.

[70] D. Foulser. Iris explorer: a framework for investigation. ACM SIGGRAPH Computer Graphics, 29(2):13-16, 1995.

[71] J. Harting, O. Mülken, and P. Borrmann. The interplay between shell effects and electron correlation in quantum dots. Phys. Rev. B, 62(15):10207, October 2000.

[72] K. Mishima, K. Yamashita, and A. Bandrauk. Isomers and transition states of the $\mathrm{Na}_{4}^{+}$clusters. ab initio studies of geometries and absorption spectra. J. Phys. Chem. A, 102(18):3157, 1998. 
[73] D. Beazley and P. Lomdahl. Lightweight computational steering of very large scale molecular dynamics simulations. In Proceedings of the 1996 ACM/IEEE conference on Supercomputing. ACM Press, 1996.

[74] The GridLab Project, http://www.gridlab.org.

[75] The Global Grid Forum, http://www.gridforum.org.

[76] SGI Inc. OpenGL Vizserver ${ }^{\mathrm{TM}}$ 3.0: Application-Transparent Remote Interactive Visualization and Collaboration. http://www.sgi.com/software/vizserver/.

[77] The Uniform Interface to Computing Resources, http://www.unicore.de.

[78] The Level 2 Grid Project, http://www.grid-support.ac.uk/12g/index.html.

[79] I. Foster and C. Kesselman. Globus: A toolkit-based grid architecture. In I. Foster and C. Kesselman, editors, The Grid: Blueprint for a New Computing Infrastructure, p 259. Morgan Kaufmann, 1999.

[80] S. Tueke, K. Czajkowski, I. Foster, J. Frey, S. Graham, C. Kesselman, D. Snelling, and P. Vanderbilt. Open Grid Services Infrastructure (OGSI) (draft). OGSI Working Group of the Global Grid Forum, 2003. http://www.gridforum.org/Meetings/ggf7/drafts/ draft-ggf-ogsi-gridservice23_2003-02-17.pdf . 
Jonathan Chin is a postgraduate student in Peter Coveney's group at the University College London. In 1999, while an undergraduate at the University of Oxford, he took a summer placement in the group, where he wrote the LB3D parallel lattice Boltzmann code and enjoyed it so much he joined the group in 2000 to do a PhD. His interests include complex fluids, visualization, and premature micro-optimization.

Jens Harting studied physics at the Carl von Ossietzky University in Oldenburg, Germany. After a Diploma thesis on Bose-Einstein Condensation he worked on path integral Monte Carlo simulations of few electron systems such as semiconductor quantum dots and received his $\mathrm{PhD}$ in December 2001. Being interested in simulations of fluids, computational steering and high performance computing, joined the RealityGrid project as a Research Fellow in Peter Coveney's group in March 2002.

Shantenu Jha is a Research Fellow with RealityGrid at the Centre for Compuatatioal Science, UCL, London. His graduate work is in Physics and Computer Science from Syracuse University, New York, USA. His interests are in computational physics, high performance and distributed computing and politics.

Peter Coveney holds a Chair in Physical Chemistry in the Department of Chemistry and is Director of the Centre for Computational Science (CCS) at University College London. His group performs internationally leading research in the area of atomistic and mesoscale modelling and simulation, including molecular dynamics, dissipative particle dynamics, lattice-gas and lattice-Boltzmann techniques and exploits state of the art high performance computing and visualisation methods. He has published numerous theoretical and modelling/simulation papers on lattice-gas and lattice-Boltzmann automata, dissipative particle dynamics and molecular dynamics inter alia. Professor Coveney is currently leading the large RealityGrid research programme, funded by the UK's Engineering and Physical Science Research Council, aimed at grid enablement of supercomputing, visualisation and computational steering (http://www.realitygrid.org). He previously held the Chair in Physical Chemistry in the Department of Chemistry at Queen Mary, University of London, before which he was with the Schlumberger Cambridge Research, where he held a number of scientific and management positions.

Andrew Porter is a software engineer in the Supercomputing, Visualization and eScience group in Manchester Computing at the University of Manchester. He graduated with a $\mathrm{PhD}$ in computational condensed-matter physics from the University of Cambridge in 2000 and, after a spell as an IT consultant in industry, joined the RealityGrid project in March 2002. His interests include computational steering and scientific visualisation. 
Stephen Pickles is Software Infrastructure Manager for the RealityGrid project, and co-leader of Manchester Computing's e-Science team at the University of Manchester. He has been engaged in grid computing since 1999. After nearly a decade as programmer and systems analyst with ICL (Australia), Stephen graduated from Macquarie University in 1994 with BSc (Hons I) in Physics, gained his PhD in lattice quantum chromodynamics from the University of Edinburgh in 1998 and then joined the CSAR service at Manchester Computing as Senior Applications Consultant. 